ORIGINAL ARTICLE

\title{
Coronary risk factors in Kawasaki disease treated with additional gammaglobulin
}

\author{
M Miura, H Ohki, T Tsuchihashi, H Yamagishi, Y Katada, K Yamada, Y Yamashita, A Sugaya, \\ O Komiyama, H Shiro
}

Arch Dis Child 2004;89:776-780. doi: 10.1136/adc.2003.032748

\begin{abstract}
See end of article for authors' affiliations

.....................

Correspondence to: Dr M Miura, Keio-Northwestern Clinic, 1701 East Woodfield Rd., \#140, Schaumburg, IL 60173, USA; miura@ as.email.ne.jp
\end{abstract}

Accepted 7 October 2003

\begin{abstract}
Aims: To assess the hypothesis that an additional intravenous gammaglobulin (IVGG) infusion, if administered early, may prevent coronary artery lesions (CAL) in patients with Kawasaki disease (KD) who do not respond to initial IVGG therapy.

Methods: Forty four KD patients (17 with CAL and 27 without CAL), treated with additional IVGG because of persistent or recrudescent fever after initial IVGG therapy, were studied. Main outcome measures were the presence of CAL by echocardiography and the number of febrile days before and after start of additional IVGG infusion (pre- and post-additional IVGG).

Results: In univariate analyses, risk factors for CAL were the number of febrile days pre-additional IVGG, the number of febrile days post-additional IVGG, the number of days that initial IVGG was divided over, the white blood cell count pre- and post-additional IVGG, and the $C$ reactive protein concentration preadditional IVGG. In a multivariate analysis, the only independent risk factor was the number of febrile days pre-additional IVGG ( $\geqslant 10$ days; odds ratio $7.86 ; 95 \% \mathrm{Cl} 1.44$ to $42.8 ; p=0.02$ ).

Conclusions: Among KD patients with persistent or recrudescent fever after initial IVGG therapy, administration of additional IVGG before the first 10 febrile days was associated with a decreased prevalence of CAL, when compared with the prevalence in those who were retreated later. An additional IVGG infusion, if administered early, may prevent CAL in initial IVGG non-responders.
\end{abstract}

A pproximately $10-20 \%$ of patients with acute Kawasaki disease $(\mathrm{KD})$ do not respond to standard intravenous gammaglobulin (IVGG) therapy and have persistent or recrudescent fever after completion of the infusion. ${ }^{1-10}$ Additional IVGG therapy is commonly used for such patients, ${ }^{1-8}$ but a preventive effect on the development of coronary artery lesions (CAL) has not been proved by retrospective studies ${ }^{1-3}$ making a comparison between KD patients treated with additional IVGG and those not treated with additional IVGG. According to the study by Burns and colleagues, ${ }^{1}$ among patients with persistent or recrudescent fever after initial IVGG therapy, CAL developed in five of 25 patients $(20 \%)$ treated with additional IVGG and none of 21 patients $(0 \%)$ not treated with additional IVGG. In the studies by Han and colleagues ${ }^{2}$ and Durongpisitkul and colleagues, ${ }^{3}$ the prevalence of CAL tended to be higher in KD patients who received additional IVGG than in those who did not receive additional IVGG (43\% $v 27 \%$ and $21 \% \vee 9 \%$, respectively). Other reports ${ }^{4-6}$ have also shown that the prevalence of CAL is high (33-54\%) in patients treated with additional IVGG. This is probably because physicians are inclined to give additional IVGG to patients who appeared more ill and were believed to be at high risk for CAL. Furthermore, we suspect that a delayed start of additional IVGG may fail to prevent CAL, since prolonged fever is a powerful predictor of $\mathrm{CAL}_{;}{ }^{11}$ however, few previous studies ${ }^{1-6}$ have referred to the number of febrile days before start of the additional IVGG infusion. We therefore focused on KD patients treated with additional IVGG owing to unresponsiveness to initial IVGG, and studied the risk factors for CAL to test a hypothesis that additional IVGG therapy, if administered early, may decrease the prevalence of CAL.

\section{METHODS}

\section{Subjects}

We reviewed the medical records of all the patients with acute typical KD at our 12 institutions between January 1993 and December 1999. Patients who received additional IVGG therapy within 14 days after completion of the initial IVGG infusion were selected for the present study. We excluded patients treated with corticosteroids, ulinastatin (a urine protease inhibitor), ${ }^{12}$ or both combined with additional IVGG to eliminate possible confounding effects. Although the additional therapies were indicated for patients who had persistent or recrudescent fever after completion of initial IVGG therapy, the decision on the administration was at the discretion of the treating physician. Serial echocardiograms were performed at admission, and one or two times a week until the 30th illness day.

\section{Data}

Medical record review was performed to extract demographic data, the course of fever, treatments received, laboratory data, and echocardiographic findings. Fever was defined as body temperature of $38.0^{\circ} \mathrm{C}$ or higher, ${ }^{4}{ }^{10}$ and the number of febrile days was calculated for periods pre-initial, preadditional, and post-additional IVGG therapies. The periods both pre-initial and pre-additional IVGG covered days from the onset of fever to the starting day of each IVGG infusion. The period post-additional IVGG covered the day following the starting day of the additional IVGG infusion to the day 14 days later. If patients had recrudescent fever, we counted only febrile days excluding afebrile days. For both initial and additional IVGG, we analysed the number of illness days at the start of infusion, the dose per body weight, and the number of days that the dose was divided over. For laboratory data, we assessed both the white blood cell count and the $\mathrm{C}$ reactive protein concentration pre-initial, preadditional, and post-additional IVGG therapies. We designated the data both pre-initial and pre-additional IVGG as those taken on either the starting day of each IVGG infusion

Abbreviations: CAL, coronary artery lesions; IVGG, intravenous gammaglobulin; KD, Kawasaki disease 
or the previous day. We designated the data post-additional IVGG as those taken during a period between the 2nd day and the 5th day after the starting day of the additional IVGG infusion. When we obtained laboratory data two or more times during these periods, we adopted the later data for preinitial and pre-additional IVGG and the earlier data for postadditional IVGG.

The subjects were divided into two groups according to the presence or absence of CAL determined by echocardiography after about one month of illness, or $30 \pm 5$ illness days. CAL were defined as the internal lumen diameter greater than two standard deviations above the expected mean calculated for body surface area on the basis of the study by De Zorzi and colleagues. ${ }^{13}$

\section{Statistics}

The relation between the variables considered as possible risk factors and the presence of CAL was examined by univariate logistic regression analyses. Continuous variables were transformed into categorical variables by the cut-off points that were defined using receiver operating characteristic curves. Six months of age was also used for the cut-off point of age. ${ }^{14}{ }^{15}$ Variables found to be associated with the presence of CAL in the univariate analyses were subjected to multivariate logistic regression analyses. All analyses were performed using SPSS, version 11.0 (SPSS Inc., Chicago, IL).

\section{RESULTS}

Of 816 patients with acute typical KD in our 12 hospitals in Japan, 746 received initial IVGG $(1-2 \mathrm{~g} / \mathrm{kg})$ administration plus oral aspirin (30-50 mg/kg/day). Brands used for IVGG were freeze dried sulphonated product (Venilon), polyethylene glycol treated product (Venoglobulin IH or Glovenin I), and PH4 treated acidic product (Polyglobin N). A total of 81 $(10.9 \%)$ patients were treated with additional therapies because of persistent fever $(n=65)$ or recrudescent fever $(n=16)$ after completion of the initial IVGG infusion. The fractions of the patients were not significantly different in any factors of initial IVGG infusion such as the number of illness days at the start, the dose, and the number of days that the dose was divided over (table 1). For additional therapies, 48 patients were treated with IVGG $(0.5-2 \mathrm{~g} / \mathrm{kg})$, 24 with IVGG combined with corticosteroids ( $1-2 \mathrm{mg} / \mathrm{kg} /$ day), ulinastatin ( $15000 \mathrm{U} / \mathrm{kg} /$ day), or both, and nine with corticosteroids or ulinastatin. Furthermore, a third course of treatment was given to nine patients: IVGG $(n=1)$, IVGG with ulinastatin $(n=5)$, corticosteroids $(n=1)$, and corticosteroids with ulinastatin $(n=2)$. Overall, we gave additional IVGG without corticosteroids or ulinastatin to 46 patients, two of whom were excluded from the study because they received it more than 14 days after completion of the infusion of initial IVGG. Of 44 patients who met the inclusion criteria, 17 (38.6\%) had CAL.

In univariate analyses, the significant risk factors for CAL $(p<0.05)$ were the number of febrile days pre-additional IVGG ( $\geqslant 10$ days), the number of febrile days post-additional IVGG ( $\geqslant 2$ days), the number of days that initial IVGG was divided over ( 4 or 5 days), the white blood cell count preadditional IVGG $(\geqslant 16000 / \mu \mathrm{l})$, the $\mathrm{C}$ reactive protein concentration pre-additional IVGG ( $\geqslant 9.7 \mathrm{mg} / \mathrm{dl})$, and the white blood cell count post-additional IVGG ( $\geqslant 11300 / \mu \mathrm{l})$ (table 2 ). Data regarding the white blood cell count and the $\mathrm{C}$ reactive protein concentration post-additional IVGG were unavailable in five patients, all of whom had no CAL. The number of illness days at the start of initial IVGG infusion was same as the number of febrile days pre-initial IVGG in all patients. The number of illness days at the start of additional IVGG infusion ( $\geqslant 10$ days) was less significantly associated with CAL $(p=0.008)$ in comparison with the number of febrile days pre-additional IVGG ( $p=0.002)$; hence, the number of febrile days pre-additional IVGG, rather than the number of illness days at the start of additional IVGG infusion, was subjected to multivariate analyses.

We performed a multiple logistic regression analysis for the variables significantly associated with the risk for CAL, excluding or including the white blood cell count postadditional IVGG separately because of unavailable data (table 3). In both exclusion and inclusion of the white blood cell count post-additional IVGG, the only independent risk factor was the number of febrile days pre-additional IVGG $(\geqslant 10$ days $)$.

Of 17 patients with CAL, none was found to have CAL before the start of the initial IVGG infusion. CAL were detected in 14 patients ( 13 males and one female; age range 1-91 months) after start of the initial IVGG infusion before start of the additional IVGG infusion. Among the 14 patients, additional IVGG was given to three before 10 febrile days and to 11 on or after 10 febrile days. CAL were detected in three patients (a 2 month old female, an 8 month old male, and a 13 month old male), after the start of the additional IVGG infusion. Additional IVGG was given to all of the three patients on or after 10 febrile days.

No adverse events were observed in any patient treated with additional IVGG. There were no signs of hypotension, congestive heart failure, allergic reaction, or hyperviscosity.

\section{DISCUSSION}

In the present study, the number of febrile days preadditional IVGG ( $\geqslant 10$ days) was an independent risk factor for CAL in patients with acute $\mathrm{KD}$ who did not respond to initial IVGG therapy and received additional IVGG therapy. It is possible that additional IVGG starting before the first 10 febrile days may prevent CAL. In this connection, let us recall the well known recommendation ${ }^{7-9}$ that initial IVGG should be given within the first 10 illness days. We believe that "febrile days", rather than "illness days", may be a better marker of the need for additional IVGG in recrudescent fever, on the basis of the result that the presence of CAL was more significantly related to the number of febrile days preadditional IVGG than the illness days at the start of the infusion of additional IVGG. Few previous reports regarding additional IVGG therapy ${ }^{1-6}$ did not refer to the timing of administration of additional IVGG; the high reported prevalence of CAL may reflect a delayed start. Prolonged fever for 10 days or longer as a risk factor for CAL is supported by the following data. (1) In a pathological study, ${ }^{16}$ the formation of CAL began after 10 illness days. (2) In an echocardiographic study, ${ }^{17}$ CAL appeared at around 10 illness days. (3) In a clinical study, ${ }^{11}$ patients with CAL had a significantly higher temperature than those without CAL during 10-13 illness days. Since additional IVGG appeared to be safe without apparent adverse effects, ${ }^{4}$ we suggest that it should be administered before the first 10 febrile days to initial IVGG non-responders.

Our results indicated that age, sex, white blood cell count, and $C$ reactive protein concentration were not independent risk factors for CAL. On the other hand, age less than 6 months ${ }^{14}{ }^{15}$ and male sex ${ }^{15}$ were reported to be risk factors for CAL in KD patients treated with IVGG. It has also been reported that CAL were associated with abnormal laboratory data pre-initial IVGG, including high counts of neutrophils and bands, ${ }^{30}$ and abnormal laboratory data post-initial IVGG, including increased white blood cell count and increased $\mathrm{C}$ reactive protein concentration. ${ }^{18}{ }^{19}$ This may be attributed to subject differences: the present study concerned only patients treated with additional IVGG, while previous studies $^{3} 1014151819$ concerned all KD patients treated with IVGG. There is a possibility that additional IVGG reduced the 
Table 1 Univariate analysis of fractions of patients treated with additional therapies in initial IVGG therapy

\begin{tabular}{|c|c|c|c|c|}
\hline & $\begin{array}{l}\text { No. of patients } \\
\text { treated with initial } \\
\text { IVGG }\end{array}$ & $\begin{array}{l}\text { No. of patients } \\
\text { treated with additional } \\
\text { therapies (\%) }\end{array}$ & $\begin{array}{l}\text { Odds ratio } \\
(95 \% \mathrm{Cl})\end{array}$ & p value \\
\hline \multicolumn{5}{|c|}{$\begin{array}{l}\text { No. of illness days at the start } \\
\text { of infusion }\end{array}$} \\
\hline$<5$ days & 226 & 29 (13) & & \\
\hline$\geqslant 5$ days & 520 & $52(10)$ & 0.91 (0.77 to 1.07$)$ & 0.25 \\
\hline \multicolumn{5}{|l|}{ Dose } \\
\hline$<1.5 \mathrm{~g} / \mathrm{kg}$ & 152 & $11(7)$ & & \\
\hline$\geqslant 1.5 \mathrm{~g} / \mathrm{kg}$ & 594 & 70 (12) & $1.20(0.96$ to 1.49$)$ & 0.11 \\
\hline \multicolumn{5}{|c|}{$\begin{array}{l}\text { No. of days that the dose was } \\
\text { divided over }\end{array}$} \\
\hline 1 or 2 days & 236 & $33(14)$ & & \\
\hline 4 or 5 days & 510 & $48(9)$ & $0.86(0.74$ to 1.01$)$ & 0.06 \\
\hline
\end{tabular}

Table 2 Univariate analysis of risk factors for coronary artery lesions

\begin{tabular}{|c|c|c|c|c|}
\hline Risk factor & $\begin{array}{l}\text { No. of } \\
\text { patients }\end{array}$ & $\begin{array}{l}\text { No. of patients } \\
\text { with CAL (\%) }\end{array}$ & Odds ratio $(95 \% \mathrm{Cl})$ & p value \\
\hline \multicolumn{5}{|l|}{ Age } \\
\hline$<6$ months & 9 & $5(56)$ & & \\
\hline$\geqslant 6$ months & 35 & $12(34)$ & $0.25(0.09$ to 1.85$)$ & 0.25 \\
\hline$<22$ months & 25 & 11 (44) & & \\
\hline$\geqslant 22$ months & 19 & $6(32)$ & $0.59(0.17$ to 2.05$)$ & 0.40 \\
\hline \multicolumn{5}{|l|}{ Sex } \\
\hline Male & 33 & $15(45)$ & & \\
\hline Female & 11 & $2(18)$ & $0.29(0.05$ to 1.43$)$ & 0.12 \\
\hline \multicolumn{5}{|c|}{ Febrile days pre-initial IVGG } \\
\hline$<5$ days & 14 & $5(36)$ & & \\
\hline$\geqslant 5$ days & 30 & $12(40)$ & $0.83(0.22$ to 3.11$)$ & 0.79 \\
\hline \multicolumn{5}{|c|}{ Febrile days pre-additional IVGG } \\
\hline$<10$ days & 22 & $3(14)$ & & \\
\hline$\geqslant 10$ days & 22 & $14(64)$ & 11.1 (2.48 to 49.5$)$ & 0.002 \\
\hline \multicolumn{5}{|c|}{ Febrile days post-additional IVGG } \\
\hline$<2$ days & 28 & $6(21)$ & & \\
\hline$\geqslant 2$ days & 16 & $11(69)$ & 8.06 (2.01 to 32.3$)$ & 0.003 \\
\hline \multicolumn{5}{|c|}{ Dose of initial IVGG } \\
\hline$<1.5 \mathrm{~g} / \mathrm{kg}$ & 4 & $2(50)$ & & \\
\hline$\geqslant 1.5 \mathrm{~g} / \mathrm{kg}$ & 40 & $15(38)$ & 0.60 (0.08 to 4.72$)$ & 0.63 \\
\hline \multicolumn{5}{|c|}{ No. of days that initial IVGG was divided } \\
\hline 1 or 2 days & 16 & $2(13)$ & & \\
\hline 4 or 5 days & 28 & $15(54)$ & $8.06(1.17$ to 41.7$)$ & 0.01 \\
\hline \multicolumn{5}{|c|}{ Dose of additional IVGG } \\
\hline$<1.5 \mathrm{~g} / \mathrm{kg}$ & 13 & $4(31)$ & & \\
\hline$\geqslant 1.5 \mathrm{~g} / \mathrm{kg}$ & 31 & $13(42)$ & $1.62(0.41$ to 6.45$)$ & 0.69 \\
\hline \multicolumn{5}{|c|}{$\begin{array}{l}\text { No. of days that additional IVGG was } \\
\text { divided over }\end{array}$} \\
\hline 1 or 2 days & 26 & $8(31)$ & & \\
\hline 4 or 5 days & 18 & $9(50)$ & 2.25 (0.65 to 7.81$)$ & 0.20 \\
\hline \multicolumn{5}{|c|}{ White blood cell count pre-initial IVGG } \\
\hline$<11300 / \mu l$ & 17 & $5(29)$ & & \\
\hline$\geqslant 11300 / \mu l$ & 27 & $12(44)$ & $1.92(0.53$ to 6.98$)$ & 0.32 \\
\hline \multicolumn{5}{|c|}{ CRP pre-initial IVGG } \\
\hline$<12.2 \mathrm{mg} / \mathrm{dl}$ & 24 & $9(38)$ & & \\
\hline$\geqslant 12.2 \mathrm{mg} / \mathrm{dl}$ & 20 & $8(40)$ & 1.11 (0.33 to 3.78$)$ & 0.87 \\
\hline \multicolumn{5}{|c|}{ White blood cell count pre-additional IVGG } \\
\hline$<16000 / \mu \mathrm{l}$ & 24 & $4(17)$ & & \\
\hline$\geqslant 16000 / \mu \mathrm{l}$ & 20 & $13(65)$ & $9.28(2.26$ to 38.1$)$ & 0.002 \\
\hline \multicolumn{5}{|c|}{$C$ reactive protein pre-additional IVGG } \\
\hline$<9.7 \mathrm{mg} / \mathrm{dl}$ & 26 & $6(23)$ & & \\
\hline$\geqslant 9.7 \mathrm{mg} / \mathrm{dl}$ & 18 & $11(61)$ & $5.24(1.41$ to 19.6$)$ & 0.01 \\
\hline \multicolumn{5}{|c|}{ White blood cell count post-additional } \\
\hline \multicolumn{5}{|l|}{ IVGG* } \\
\hline$<11300 / \mu l$ & 22 & $6(27)$ & & \\
\hline$\geqslant 11300 / \mu \mathrm{l}$ & 17 & $11(65)$ & 4.88 (1.25 to 19.2$)$ & 0.02 \\
\hline \multicolumn{5}{|c|}{$C$ reactive protein post-additional IVGG* } \\
\hline$<5.2 \mathrm{mg} / \mathrm{dl}$ & 22 & $7(32)$ & & \\
\hline$\geqslant 5.2 \mathrm{mg} / \mathrm{dl}$ & 17 & $10(59)$ & $3.06(0.82$ to 11.4$)$ & 0.10 \\
\hline
\end{tabular}


Table 3 Multivariate analysis of risk factors for coronary artery lesions

\begin{tabular}{|c|c|c|c|c|}
\hline \multirow{2}{*}{$\begin{array}{l}\text { Risk factor (cut-off point } \\
\text { for categorical variable) }\end{array}$} & \multicolumn{2}{|c|}{$\begin{array}{l}\text { Exclusion of white blood cell } \\
\text { count post-additional IVGG } \\
\text { ( } n=44)\end{array}$} & \multicolumn{2}{|c|}{$\begin{array}{l}\text { Inclusion of white blood cell coun } \\
\text { post-additional IVGG } \\
(\mathrm{n}=39)\end{array}$} \\
\hline & Odds ratio $(95 \% \mathrm{Cl})$ & $\mathrm{p}$ value & Odds ratio $(95 \% \mathrm{CI})$ & p value \\
\hline $\begin{array}{l}\text { Febrile days pre-additional IVGG } \\
(<10 \text { days } / \geqslant 10 \text { days })\end{array}$ & 7.86 (1.44 to 42.8$)$ & 0.02 & 25.6 (2.31 to 289.1$)$ & 0.008 \\
\hline $\begin{array}{l}\text { Febrile days post-additional IVGG } \\
(<2 \text { days } / \geqslant 2 \text { days })\end{array}$ & $4.42(0.82$ to 23.8$)$ & 0.08 & $5.90(0.90$ to 38.9$)$ & 0.07 \\
\hline $\begin{array}{l}\text { Number of days that initial IVGG was } \\
\text { divided over ( } 1 \text { or } 2 \text { days } / 4 \text { or } 5 \text { days) }\end{array}$ & $3.40(0.44$ to 26.3$)$ & 0.24 & $3.16(0.44$ to 29.2$)$ & 0.31 \\
\hline $\begin{array}{l}\text { White blood cell count pre-additional } \\
\text { IVGG }(<16000 / \mu \mathrm{l} / \geqslant 16000 / \mu \mathrm{l})\end{array}$ & 4.19 (0.79 to 22.2$)$ & 0.09 & $3.00(0.38$ to 23.9$)$ & 0.30 \\
\hline $\begin{array}{l}\text { C reactive protein pre-additional IVGG } \\
(<9.7 \mathrm{mg} / \mathrm{dl} / \geqslant 9.7 \mathrm{mg} / \mathrm{dl})\end{array}$ & $1.71(0.20$ to 14.7$)$ & 0.63 & $1.79(0.15$ to 21.1$)$ & 0.64 \\
\hline $\begin{array}{l}\text { White blood cell count post-additional } \\
\text { IVGG }(<11300 / \mu \mathrm{l} / \geqslant 11300 / \mu \mathrm{l})\end{array}$ & - & - & 9.35 (0.93 to 94.3$)$ & 0.06 \\
\hline
\end{tabular}

risk factors for CAL in initial IVGG non-responders, such as age $<6$ months, male sex, high white blood cell count, and high $\mathrm{C}$ reactive protein concentration in the present study.

In order to start the infusion of additional IVGG before the first 10 febrile days, we make three recommendations to physicians who treat patients with acute KD. First, KD patients should be treated immediately with an initial IVGG infusion. Although the efficacy of early treatment before five days of illness is controversial, ${ }^{20}$ Tse and colleagues ${ }^{21}$ reported that treatment with initial IVGG on or before the fifth illness day resulted in better coronary outcome. Second, initial IVGG should be given in a single infusion, because 4-5 daily infusions led to delayed administration of additional IVGG. In Japan, the medical insurance system previously prevented physicians from treating with a single infusion despite the proven efficacy, ${ }^{9}$ but it has recently allowed its use. Third, it should be judged early whether patients respond to initial IVGG or not. However, we agree with Burns and colleagues ${ }^{1}$ and Brogan and colleagues ${ }^{7}$ that patients with fever at least 48 hours after completion of a single infusion of initial IVGG are considered non-responders, since occasional responders have fever during the period. If additional IVGG is given 48 hours after completion of the infusion before the first 10 febrile days, initial IVGG should be completed by the first seven febrile days.

The present study has some limitations. There may be unknown selection bias owing to the retrospective nature of the study. A prospective randomised control study would be optimal to establish the efficacy of additional IVGG for patients who do not respond to initial IVGG, but it would be difficult to justify inclusion of an untreated control group in view of the increased risk of CAL in patients with persistent or recrudescent fever. We used four or five daily infusions of IVGG, which is not currently recommended in other countries..$^{7-9}$ The administration of various brands of IVGG may be a confounding factor. ${ }^{20}$

In our study, among KD patients with persistent or recrudescent fever after initial IVGG therapy, administration of additional IVGG before the first 10 febrile days was associated with a decreased risk for CAL, when compared with the risk in those who were retreated later. This finding provides support for early diagnosis and initial treatment of $\mathrm{KD}$ and suggests that an additional IVGG infusion, if administered early, may prevent CAL in initial IVGG nonresponders.

\section{ACKNOWLEDGEMENTS}

We are grateful to Dr Anne $\mathrm{H}$ Rowley of the Departments of Pediatrics and Microbiology/Immunology at Northwestern University Feinberg School of Medicine, and Dr Mitio Inokuti of Argonne
National Laboratory for critical reading of an earlier manuscript. We are indebted to Dr Hiroshi Yanagawa of Saitama Prefectural University and Dr Yosikazu Nakamura of the Department of Public Health at Jichi Medical School for providing data of nationwide epidemiologic surveys on the incidence of Kawasaki disease in Japan. Furthermore, we would like to thank T Takahashi, Y Kojima, M Satoh, T Murai, M Ihara, J Ishihara, M Tokumura, H Toyoma, T Furuta, H Fukushima, J Maeda, E Takahashi, H Ueda, and S Yoshiba at Keio University School of Medicine for their excellent cooperation. The following institutions participated in the present study: Keio University School of Medicine, Tokyo; Tokyo Metropolitan Kiyose Children's Hospital, Tokyo; Tokyo Metropolitan Ohtsuka Hospital, Tokyo; Kawasaki Municipal Hospital, Kawasaki; Yokohama Municipal Citizens' Hospital, Yokohama; Yokohama Rosai Hospital, Yokohama; Saitama Municipal Hospital, Saitama; Isehara Kyodo Hospital, Isehara; Hiratsuka City Hospital, Hiratsuka; Hiratsuka Kyosai Hospital, Hiratsuka; Ashikaga Red Cross Hospital, Ashikaga; Saiseikai Utunomiya Hospital, Utunomiya.

\section{Authors' affiliations}

M Miura, H Ohki, T Tsuchihashi, H Yamagishi, Y Katada, K Yamada, Y Yamashita, A Sugaya, O Komiyama, H Shiro, Department of Pediatrics, Keio University School of Medicine, Tokyo, Japan

\section{REFERENCES}

1 Burns JC, Capparelli EV, Brown JA, for the US/Canadian Kawasaki Syndrome Study Group, et al. Intravenous gamma-globulin treatment and retreatment in Kawasaki disease. Pediatr Infect Dis J 1998;17:1144-8.

2 Han RK, Silverman ED, Newman A, et al. Management and outcome of persistent or recurrent fever after initial intravenous gamma globulin therapy in acute Kawasaki disease. Arch Pediatr Adolesc Med 2000; 154:694-9.

3 Durongpisitkul K, Soongswang J, Laohaprasitiporn D, et al. Immunoglobulin failure and retreatment in Kawasaki disease. Pediatr Cardiol 2003:24:145-8.

4 Sundel RP, Burns JC, Baker A, et al. Gamma globulin re-treatment in Kawasaki disease. J Pediatr 1993;123:657-9.

5 Hashino K, Ishii $M$, lemura $M$, et al. Re-treatment for immune globulinresistant Kawasaki disease: a comparative study of additional immune globulin and steroid pulse therapy. Pediatr Int 2001;43:211-17.

6 Wallace CA, French JW, Kahn SJ, et al. Initial intravenous gammaglobulin treatment failure in Kawasaki disease. Pediatrics 2000;105:E78.

7 Brogan P, Bose A, Burgner D, et al. Kawasaki disease: an evidence based approach to diagnosis, treatment, and proposals for future research. Arch Dis Child 2002;86:286-90.

8 Rowley AH, Shulman ST. Kawasaki syndrome. Pediatr Clin North Am 1999;46:313-29.

9 Newburger JW, Takahashi M, Beiser AS, et al. A single intravenous infusion of gamma globulin as compared with four infusions in the treatment of acute Kawasaki syndrome. N Engl J Med 1991;324:1633-9.

10 Beiser AS, Takahashi M, Baker AL, et al. For the US multicenter Kawasaki disease study group. A predictive instrument for coronary artery aneurysms in Kawasaki disease. Am J Cardiol 1998;81:1116-20.

11 Koren G, Lavi S, Rose V, et al. Kawasaki disease: review of risk factors for coronary aneurysms. J Pediatr 1986;108:388-92.

12 Zaitsu M, Hamasaki Y, Tashiro K, et al. Ulinastatin, an elastase inhibitor, inhibits the increases mRNA expression of prostaglandin $\mathrm{H} 2$ synthase-type 2 in Kawasaki disease. J Infect Dis 2000;181:1101-9. 
13 De Zorzi A, Colan SD, Gauvreau K, et al. Coronary artery dimensions may be misclassified as normal in Kawasaki disease. J Pediatr 1998; 133:254-8

14 Rosenfeld EA, Corydon KE, Shulman ST. Kawasaki disease in infants less than one year of age. J Pediatr 1995; 126:524-9.

15 Ayusawa M, Harada K. Appropriate use of $\gamma$-globulin for Kawasaki disease [in Japanese]. Shonika 1995;36:461-6.

16 Naoe S, Takahashi K, Masuda H, et al. Kawasaki disease with particular emphasis on arterial lesions. Acta Pathol Jpn 1991:41:785-97.

17 Kato H. Cardiovascular involvement in Kawasaki disease: evaluation and natural story. In: Shulman ST, ed. Kawasaki disease. New York: Alan R, Liss, 1987:277-86.
18 Mori M, Imagawa T, Yasui K, et al. Predictors of coronary artery lesions after intravenous $\gamma$-globulin treatment in Kawasaki disease. J Pediatr 2000;137:177-80.

19 Miura M, Kojima Y, Satoh M, et al. Development of criteria to determine risk of coronary artery aneurysms in patients with Kawasaki disease after intravenous gamma globulin treatment [in Japanese]. Pediatr Cardiol Cardiac Surg 1997; 13:12-18.

20 Newburger JW, Taubert KA, Shulman ST, et al. Summary and abstracts of the seventh international Kawasaki disease symposium. Pediatr Res 2003;53:153-7.

21 Tse SM, Silverman ED, McCrindle BW, et al. Early treatment with intravenous immunoglobulin in patients with Kawasaki disease. J Pediatr 2002;140:450-5.

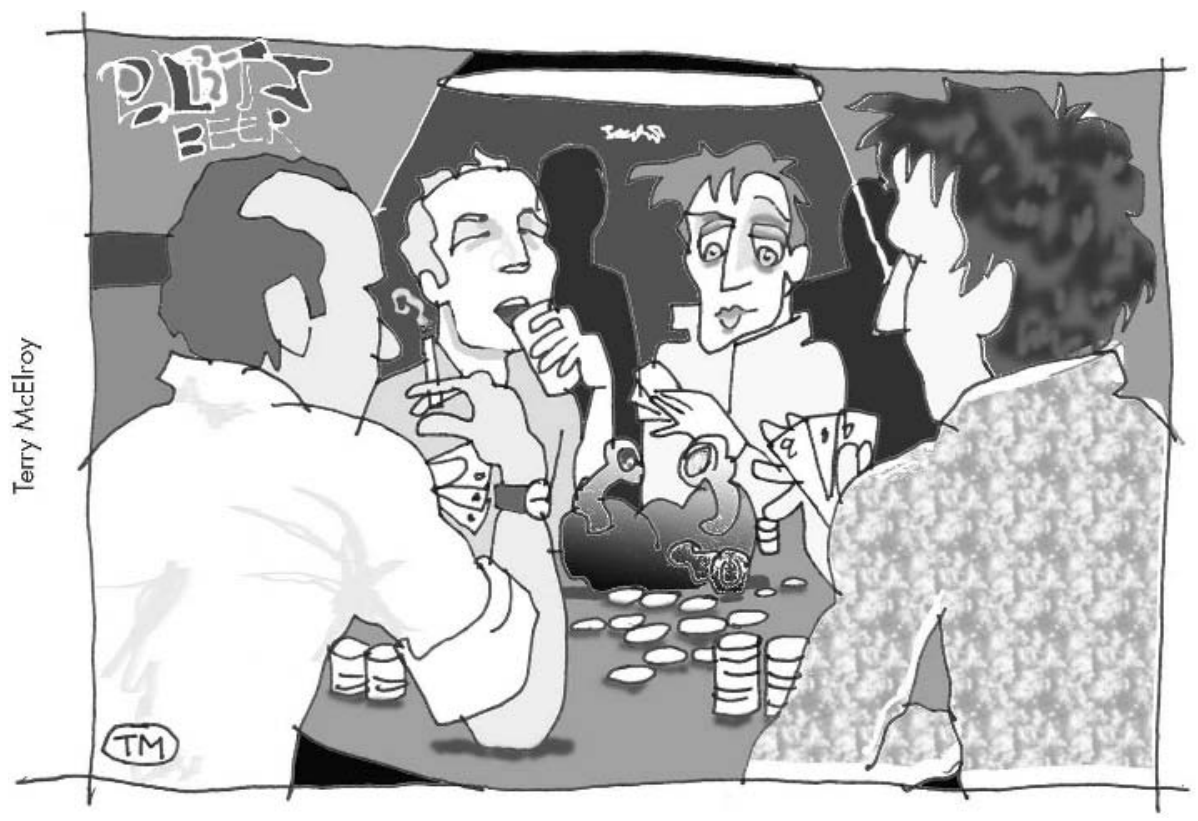

